\title{
Efeitos biológicos da luz: aplicação de terapia de baixa potência empregando LEDs (Light Emitting Diode) na cicatrização da úlcera venosa: relato de caso
}

\section{Biological effects of light: application of low power therapy using LEDs (Light Emitting Diode) on the healing of venous ulcers: a case report}

\author{
Cláudia Patrícia Cardoso Martins Siqueira ${ }^{1}$; Dari de Oliveira Toginho Filho; \\ Franciele Mendes de Lima ${ }^{3}$; Francisco Pereira Silva ${ }^{4}$; Henrique Durante ${ }^{5}$; Ivan \\ Frederico Lupiano Dias ${ }^{6}$; José Leonil Duarte ${ }^{6}$; Roberto Kiyoshi Kashimoto ${ }^{5}$; \\ Valdênea Aparecida Bordinassi de Castro ${ }^{7}$
}

\begin{abstract}
Resumo
O emprego de fontes de luz de baixa potência, como diodos emissores de luz-LEDs, pode propiciar um recurso terapêutico opcional aos convencionais ou ser utilizado em conjunto com estes, com a vantagem do baixo custo e comprovada eficiência no tratamento de úlceras e outras enfermidades. $\mathrm{O}$ estudo de caso foi realizado com um paciente do Ambulatório de Clinicas do HC/UEL que apresenta úlceras nos membros inferiores. A aplicação foi feita uma vez por semana, empregando LEDs com comprimento de onda de $628 \mathrm{~nm}$ na úlcera do membro inferior esquerdo e o direito foi utilizado como controle. A evolução foi medida por meio de registro fotográfico, medida da área e mensuração da dor. Foram realizadas 18 sessões. Os resultados apontaram mudanças nas características clínicas da lesão e a área cicatrizada foi $30 \%$ maior em relação à úlcera controle. Quanto à dor, a escala análoga visual variou de oito para zero no membro irradiado e de nove para dois no controle. Assim, podese concluir que a LED terapia é um recurso de eleição no tratamento da úlcera venosa, nos aspectos cicatriciais e redução da dor.
\end{abstract}

Palavras-chave: Úlcera. LED. Cicatrização.

\begin{abstract}
The use of low power light sources, such as diodes emitters of light - LEDs, provides an alternative and complementary therapeutic resource to the conventional ones, with the advantage of being cost-effective and reliable in the treatment of ulcers and other infirmities. This study was carried out on a patient from the HC/UEL Outpatient Clinic with ulcers on both lower limbs. LEDs were applied once a week, using a wave length of $628 \mathrm{~nm}$ on the left lower limb ulcer ,using the right limb as control. Evolution
\end{abstract}

\footnotetext{
1 Fisioterapeuta formada pela UEL, Aprimoramento em Ortopedia e Traumatologia pela USP - Ribeirão Preto, Especialista em Ciências Fisiológicas - UEL - Londrina, Doutora em Bases Gerais da Cirurgia - UNESP - Botucatu, Docente do Curso de Fisioterapia da UEL. E-mail: claudia@uel.br.

2 Professor Adjunto Temporário e Físico do Departamento de Física da UEL.

3 Aluna de Graduação de Física da UEL e Bolsista da Fundação Araucária.

4 Professor Associado do Departamento de Clinica Cirúrgica da UEL.

5 Residentes em Fisioterapia Traumato-Ortopédica Funcional do HU-UEL.

6 Professor Associado do Departamento de Física da UEL.

Enfermeira Chefe do Setor 1 do HC-UEL.
} 
was measured through photographic registers, area size and pain assessment. Eighteen sessions were performed. Results showed that changes were observed on the clinical characteristics of the injury and the healed area was 30\% larger when compared to the control. As for the pain assessment, the visual analogue scale varied from eight to zero on the limb radiated and from nine to two on the control. Thus, it can be concluded that the LED therapy is a resource of choice for treating venous ulcer, in both healing and pain reduction aspects.

Key words: Ulcer. LED. Healing.

\section{Introdução}

Até o início da década de 80 , desconheciam-se os mecanismos da radiação eletromagnética em nível molecular e celular (KARU, 1987). Trabalhos científicos realizados ao longo da década de 80 (KARU et al., 1982, 1983) estabeleceram as bases para a compreensão dos mecanismos moleculares associados aos efeitos da luz sobre as células.

Além disso, a resposta celular à fotoestimulação não está associada a propriedades específicas da luz LASER, como a coerência. Isto permitiu o trabalho com fontes emissoras de luz não coerentes como os diodos emissores de luz - LEDs. Estes dispositivos são mais baratos, de maior facilidade de manuseio, e operam com correntes elétricas relativamente baixas em comparação aos LASERs (SCHUBERT, 2006).

O emprego de LEDs e/ou LASERs de baixa potência com finalidades terapêuticas vem sendo muito ampliado ao longo dos últimos anos com farta literatura disponível em sites e em diversas revistas científicas. A foto estimulação causada pela irradiação por LEDs ou LASERs de baixa potência apresenta uma série de resultados tais como, entre outros, o aumento da taxa de proliferação de células (KARU, 1987), o aumento da taxa de produção de fibroblastos e da síntese de colágenos (TAKAHASHI et al., 1992; VINCK et al., 2003), aumento da taxa de síntese de RNA e DNA (KARU, 1987; KARU; KOLYAKOV, 2005), síntese de ATP (YU et al., 1997), aumento da vascularização (CONLAN; RAPLEY; COBB, 1996), variações na condução nervosa (VINCK et al., 2005).

A aplicação do LED como recurso terapêutico vem se ampliando na última década. Estudos desenvolvidos pela NASA (National Aero Space Agency) nos Estados Unidos demonstraram fortes evidências de que a eficiência dos LEDs em processos de foto estimulação celular é semelhante à dos LASERs de baixa potência - terapia LLLT (VINCK et al., 2003). Testes clínicos com aplicação de LEDs em cicatrização de úlceras em seres humanos já foram liberados pela FDA (Food Drug Administration) norte-americana (WHELAN et al., 2001). Trabalhos recentes desenvolvidos no Brasil demonstram a eficiência deste recurso para o tratamento de diversos tipos de úlceras (CORAZZA, 2005; MARQUES, 2004).

A úlcera de membros inferiores é caracterizada por perda circunscrita ou irregular do tegumento (derme e hipoderme), geralmente relacionada ao sistema vascular, arterial ou venoso (FRADE et al., 2005). A úlcera venosa é a complicação mais importante da insuficiência venosa crônica e representa 60 a $70 \%$ de todas as úlceras de membro inferior (MAFFEI et al., 2002).

A incidência das úlceras de membros inferiores na Europa fica entre $0,18 \%$ e $1 \%$. Ao se projetar esta incidência para o Brasil, com 160 milhões de habitantes, este número fica em torno de 1,5 milhões de pessoas. As despesas com o tratamento destes pacientes são muito altas, tendo sido estimado que, durante quatro meses de tratamento ambulatorial, avaliando o material gasto nos curativos, elas variaram entre 25 e 2.500 dólares (THOMAZ, 2002).

O tratamento, geralmente de caráter ambulatorial, requer consultas freqüentes ao médico, com comprometimento da qualidade de vida do paciente. Em número reduzido de casos, torna-se necessário 
efetuar o tratamento no âmbito hospitalar. As taxas de cicatrização são, em geral, baixas e as recidivas são freqüentes (THOMAZ, 2002).

Entre os recursos terapêuticos empregados para o tratamento de úlceras, além dos tratamentos tradicionais com medicação e compressão, podem ser citados a Criogenia, O Ultra-Som, Eletroterapia (CORRÊA, 2003), Oxigenoterapia Hiperbárica (DIAS, 2001), Laser de baixa potência (CHAVANTES; VASEY; KUALE, 1998) e ou o emprego conjugado destes recursos como a Oxigenoterapia Hiperbárica com LED (MARQUES, 2004).

Apesar da publicação de diversas recomendações de tratamento e das revisões sistemáticas realizadas, o tratamento adequado das úlceras venosas da perna é desafio contínuo para os profissionais de saúde (THOMAZ, 2002). É fundamental a continua implementação de equipes multidisciplinares e estudos bem elaborados com recomendações baseadas na evidência e amplamente divulgadas para maximizar os benefícios a estes doentes. O objetivo deste trabalho foi analisar a aplicação do LED na evolução cicatricial e dor em individuo portador de ulcera em ambos os membros inferiores.

\section{Material e métodos}

Sujeito: Indivíduo, 86 anos, masculino, branco, aposentado, com diagnóstico de úlcera venosa de estase em membros inferiores, sem doença arterial e diabetes. A úlcera do membro inferior direito estava presente desde 28/05/07 e no membro inferior esquerdo desde 1987. Após admissão do paciente, o mesmo foi submetido a uma avaliação clínica (dados pessoais, característica da lesão, tratamentos prévios e doenças associadas) realizado pelos autores da pesquisa.

Considerações éticas: A presente pesquisa foi aprovada pelo Comitê de Ética em pesquisa da UEL de acordo com o parecer 315/06 de 02/02/07. $\mathrm{O}$ indivíduo foi esclarecido sobre a natureza da pesquisa e assinou o termo de consentimento livre e esclarecido.

Procedimento: Os processos de avaliação e tratamento foram realizados após o desbridamento das úlceras durante as 18 sessões. Ao ser admitido na sala de consulta, o indivíduo foi posicionado sentado com apoio em terço superior da perna, deixando a úlcera livre e após foram realizados retirada do curativo e desbridamento.

Logo após, o mesmo foi posicionado em decúbito dorsal, com um campo estéril sob os membros inferiores, em seguida, foram realizadas fotografias digitais (LAGAN et al., 2002) da área ulcerada bilateral com máquina fotográfica digital ${ }^{8}$ a uma distância de 20 centímetros, nas seguintes posições: membro inferior rodado externamente, internamente e na posição neutra.

Para a mensuração da área da ferida, foi utilizado um protetor plástico estéril, posicionado sobre a úlcera e foi delimitada a borda interna da ferida com pincel $^{9}$ de ponta fina. Após, este plástico foi posicionado sobre um anteparo de papel e a área da ferida foi transferida para o papel vegetal. Este desenho foi escaneado por meio de um software de digitalização ${ }^{10}$ e sua área foi mensurada pelo programa de cálculo ${ }^{11}$. A dor foi avaliada no início da sessão por meio da Escala Visual Análoga (EVA) (LAGAN et al., 2002).

Foi montado no Laboratório de Óptica e Optoeletrônica do Departamento de Física da UEL, um dispositivo, contendo sete LEDs de alto brilho ${ }^{12}$ (Figura 1) com emissão no comprimento de onda de 625 nm (MARQUES et al., 2004). A alimentação do dispositivo óptico foi feita por uma fonte de corrente elétrica constante, calibrada para permitir doses de luz de quatro $\mathrm{J} / \mathrm{cm}^{2}$ com um "spot" de aproximadamente dois $\mathrm{cm}^{2}$ de área.

\footnotetext{
8 Sony ${ }^{\circledR}$ Cyber-Shot, modelo DSC - 5700.

$9 \operatorname{moLin} \AA$.

10 Windig ${ }^{\circledR} 2.5$.

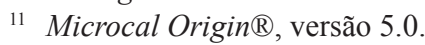

12 OptoSupply.
} 
A aplicação do LED foi feita no membro inferior esquerdo e o membro inferior direito foi eleito como controle do tratamento. O terapeuta e o paciente utilizaram óculos de proteção ${ }^{13}$. O dispositivo LED foi posicionado a 90 graus e a uma distância de dois cm sobre os cinco pontos mostrados na Figura 2 durante dois minutos em cada ponto. Após a aplicação do LED, o curativo foi realizado conforme a rotina do setor. Este paciente fez uso de faixa elástica em membros inferiores diariamente.

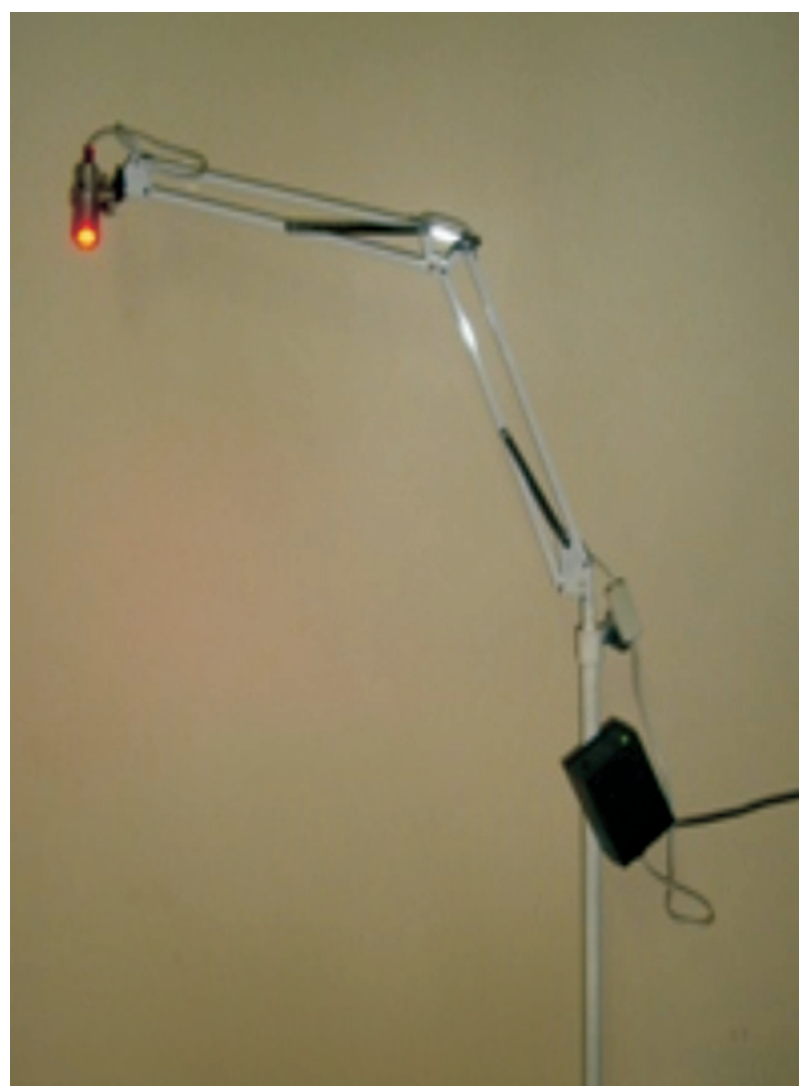

Figura 1. Equipamento contendo o dispositivo com LEDs, desenvolvido pelo Laboratório de Óptica e Optoeletrônica da UEL.

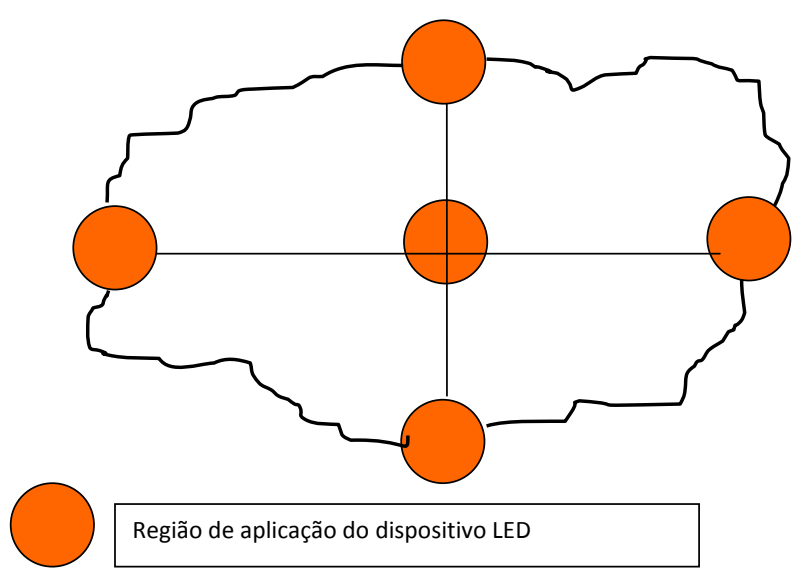

Figura 2. Esquema gráfico da úlcera com a demarcação dos pontos irradiados.

\section{Resultados}

Quanto aos aspectos clínicos, na úlcera do membro inferior esquerdo após as 18 aplicações do LED, foram observados: diminuição da profundidade, desaparecimento da área de necrose em região lateral, surgimento de ilhas de cicatrização no meio e bordas, diminuição do exsudato, coloração mais clara do leito da ferida; porém uma nova lesão surgiu na parte superior da ferida (Figura 3).

Em relação ao membro inferior direito, que foi tomado como controle, pode se observar: leve redução da profundidade da lesão, separação da ferida em duas partes de menor área, cor do leito pouco mais escuro em relação ao membro inferior esquerdo, desaparecimento da área de necrose em região lateral da ferida, porém aparecimento de área de necrose em ferida medial (Figura 4).

${ }^{13}$ MM Optics ${ }^{\circledR}$. 


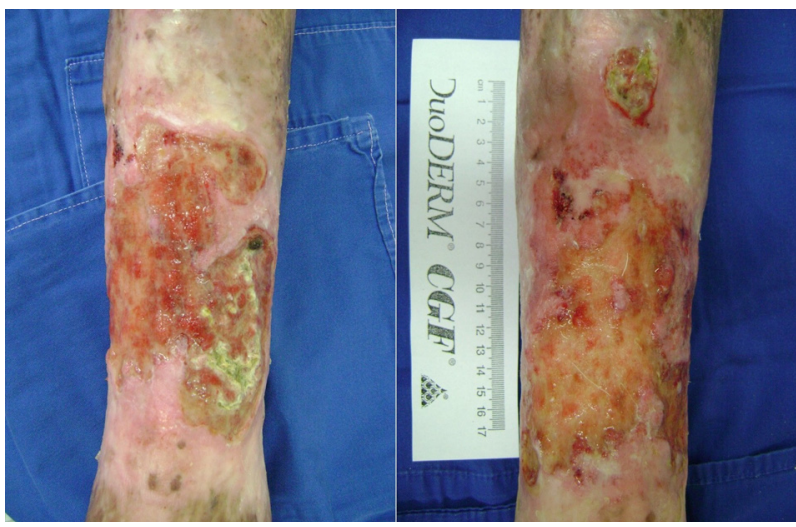

Figura 3. Aspectos Clínicos da Úlcera do membro inferior esquerdo (irradiado), na primeira e última sessão, respectivamente.

Com relação à área das úlceras, logo após a primeira sessão de tratamento, pôde-se verificar leve aumento na área de ambas as úlceras e, nas sessões subseqüentes, ocorreu um decréscimo até a décima segunda sessão. Entre a décima segunda e décima oitava, ocorreu discreto aumento na área das úlceras, conforme Figura 5.

Ao se avaliar a área cicatrizada, pode se observar que a úlcera com aplicação do LED evoluiu de forma diferenciada em relação à área da úlcera controle.

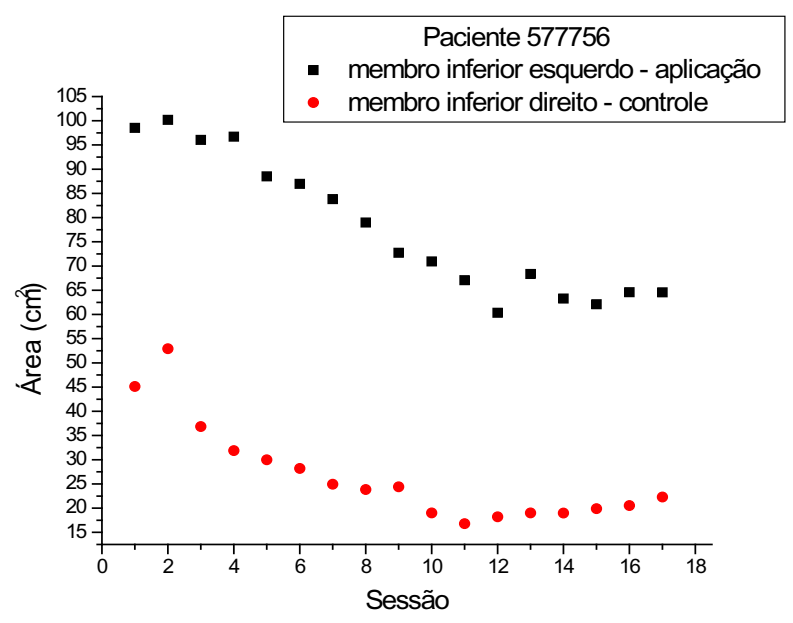

Figura 5. Área das úlceras ao longo do período de tratamento.

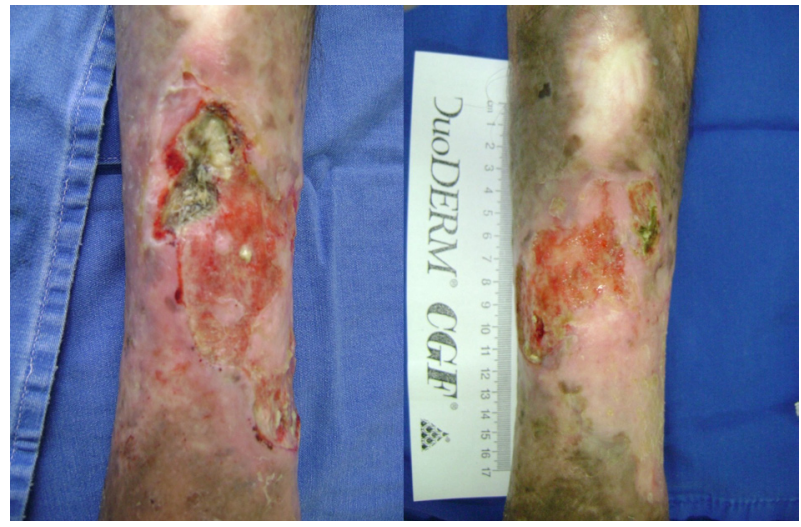

Figura 4. Aspectos Clínicos da Úlcera do membro inferior direito (controle), primeira e última sessão, respectivamente.

A área cicatrizada da úlcera controle aumentou até a décima segunda sessão e depois diminuiu. A área cicatrizada da úlcera com aplicação do LED continuou a aumentar estabilizando-se em um patamar nitidamente superior $\left(32,5 \mathrm{~cm}^{2}\right)$ em relação à área da úlcera controle (aproximadamente 22,5 $\mathrm{cm}^{2}$ ). Na décima oitava sessão, a área cicatrizada da úlcera irradiada foi aproximadamente $30 \%$ maior em relação à área cicatrizada da úlcera controle (Figura 6).

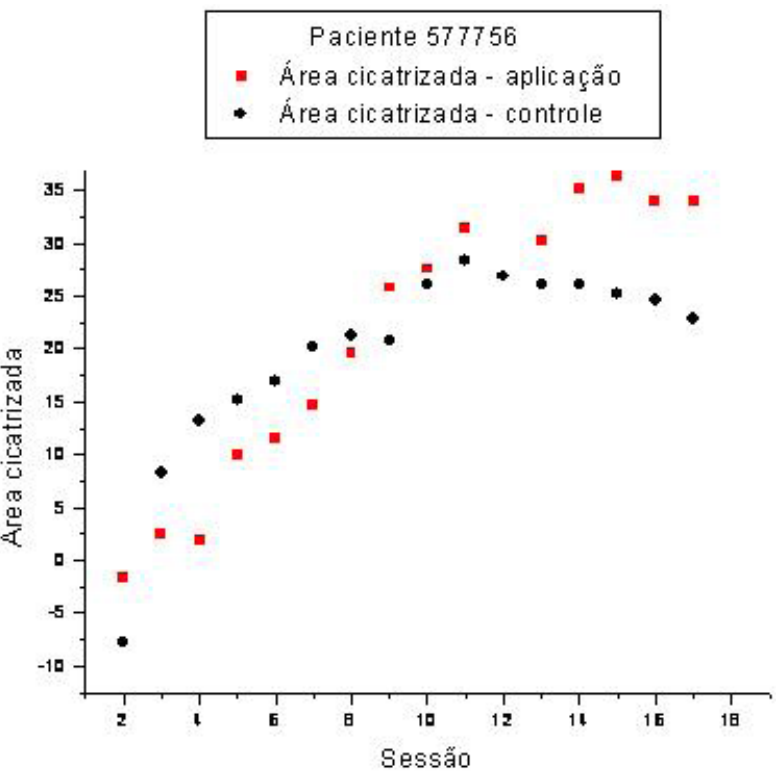

Figura 6. Evolução das áreas de cicatrização das úlceras. 
Ambos os membros mostram um quadro similar de dor antes do início do tratamento, decrescendo após as sessões. A evolução da dor é nitidamente diferenciada, já nas primeiras aplicações do LED, decrescendo de modo acentuado e atingindo um valor nulo, já na segunda sessão e oscilando a seguir, porém sempre em um patamar médio inferior quando comparado à dor no membro inferior direito (Figura 7).

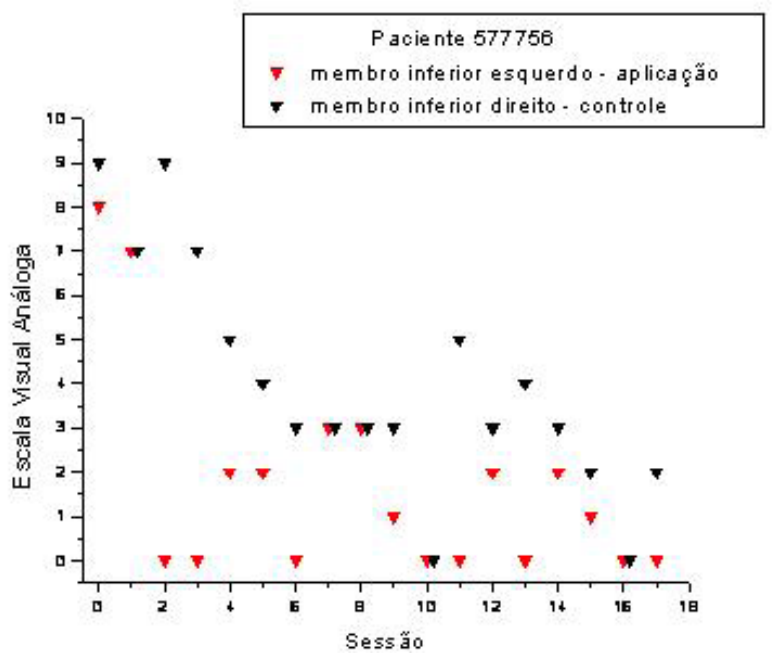

Figura 7. Evolução do quadro de dor antes do tratamento e após as aplicações do LED.

\section{Discussão}

Poucos são os artigos que descrevem as características clinicas das úlceras venosas, pela dificuldade em realizar uma avaliação quantitativa das mesmas. Considerando estes aspectos, justificase a importância da avaliação clínica, uma vez que a técnica do decalque não fornece informações relevantes como profundidade e aparência das lesões (BRASIL, 2002). A partir desta avaliação, é possível ao pesquisador utilizar destes dados para confirmar e complementar seu estudo, apresentando maior fidedignidade ao mesmo.

O exame detalhado da região estudada e o acompanhamento da evolução do tratamento são fatores importantes que auxiliam na avaliação da cicatrização. No presente estudo, do ponto de vista clínico, o membro inferior esquerdo, após a aplicação do LED, obteve redução importante na profundidade da lesão, surgimento de ilhas de cicatrização e coloração mais clara do leito da ferida. O membro inferior direito (controle), apesar de também apresentar diminuição da profundidade, esta não foi tão evidente quanto no membro contralateral, e a cor do leito da ferida passou a ser mais escura.

Lagan et al. (2002) também utilizou avaliação clinica, com descrição qualitativa do aspecto da ferida realizada sempre por um mesmo pesquisador, sendo coletados dados detalhados da aparência da ferida a cada intervenção. De forma semelhante, a presente pesquisa utilizou um questionário a cada sessão abordando detalhes da úlcera, como a característica do exsudato, cor do leito da ferida, entre outros.

Lagan et al. (1999) observou que as fotografias das úlceras não tinham confiabilidade para mensuração das feridas, por isso este estudo não as utilizou com este objetivo, mas sim de promover dados complementares no aspecto das mesmas. Apesar da subjetividade deste método de avaliação, este parâmetro associado a outras técnicas de mensuração, pode tornar mais fidedigno os resultados do tratamento.

A cicatrização das feridas é um processo complexo que inclui uma seqüência coordenada de alterações bioquímicas, envolvendo as interações de vários tipos diferentes de células, os componentes da matriz, as proteases e citocinas (REDDY, 2006). Com o objetivo de reduzir possíveis erros na medida da úlcera, foi utilizado o método decalque, pois a maior parte dos artigos utiliza medição por fita métrica, o que torna a medida não reprodutiva e menos confiável (LAGAN et al., 2002). Na presente pesquisa, foi observado aumento da área ulcerada no inicio do tratamento, porém com a evolução do tratamento, a área da úlcera teve evolução semelhante a da área não irradiada.

Quanto à área cicatrizada, o membro controle estabilizou após a $12^{\mathrm{a}}$ sessão, diferente da área 
cicatrizada do membro irradiado que continuou aumentando após este tempo, o que parece mostrar um efeito tardio do LED no processo de reparação cicatricial. Apesar de ter utilizado métodos diferentes, Marques et al. (2004) também observaram melhora da cicatrização tecidual e redução da ulcera em todos os pacientes.

Yu et al. (1997) em experimentos com ratos diabéticos, investigaram a eficácia do laser com comprimento de onda semelhante ao da presente pesquisa associado ou não ao fator de crescimento e observaram melhora na cicatrização nos grupos que usaram o laser.

A úlcera venosa é a mais freqüente seqüela da insuficiência venosa e afeta negativamente a qualidade de vida desses pacientes, sendo a dor uma das principais causas deste problema (GUARNERA et al., 2007; HERBER; SCHNEPP; RIEGER, 2007). Mesmo assim, este importante sintoma é negligenciado durante o tratamento da mesma (BARRON; JACOB; KIRSNER, 2007). Alguns métodos que, além de facilitar a cicatrização, possam promover analgesia estão sendo aplicados como os curativos com géis, as faixas de compressão elástica (FRANKS et al., 2007; JORGENSEN; FRIIS; GOTTRUP, 2006) e, dentre outros, o laser de baixa potencia que ainda necessita de maiores estudos para comprovar sua eficácia no tratamento da dor em pacientes com úlcera venosa (LAGAN et al., 2002); assim como, o mesmo já comprovou seus efeitos no tratamento de outras algias (JENSEN; HARMS-RINGDAHL，2007; LAM; CHEING, 2007; STERGIOULAS, 2008).

Lagan et al. (2002), utilizando laser de baixa potência e fototerapia, relata possível melhora da dor, após doze semanas de tratamento, diferentemente deste estudo, no qual a dor chega a zero na segunda sessão e oscila até a $18^{\mathrm{a}}$ sessão estabilizando em um patamar inferior ao da úlcera controle; o que também foi encontrado por Lagan et al. (2000) em um estudo de caso sobre o laser em úlceras venosas.
Lagan et al. (2001) relata a ineficiência de um dispositivo laser para o controle da dor, porém em incisões cirúrgicas nos pés, assim como Gaida et al. (2004) cita que existem evidências limitadas de que o laser de baixa potência apresenta influência sobre a dor de pacientes com queimaduras; o que, apesar de não serem estudos em úlceras venosas, demonstram que a terapia com luz de baixa potência ainda precisa de maiores estudos que comprovem a sua eficácia.

As conclusões obtidas abrem novas perspectivas para futuros estudos que associem o LED a outros tipos de intervenção, pois a aplicação do mesmo parece apresentar efeitos benéficos na cicatrização e dor, o que pode melhorar a qualidade de vida do paciente.

\section{Conclusão}

$\mathrm{Na}$ presente pesquisa, a aplicação de luz empregando LEDs, com comprimento de onda de $625 \mathrm{~nm}$, em indivíduo portador de úlcera em ambos os membros inferiores, mostra a potencialidade desta alternativa de tratamento na evolução cicatricial e redução da dor. Um outro fator a se considerar no presente é trabalho é o custo extremamente baixo do equipamento fabricado no Laboratório de Física da UEL, em comparação aos encontrados no mercado, o que pode viabilizar sua aplicação em escala que traga benefícios às camadas menos favorecidas da população.

\section{Referências}

BARRON, G. S.; JACOB, S. E.; KIRSNER, R. S. Dermatologic complications of chronic venous disease: medical management and beyond. Annals of Vascular Surgery, Cambridge, v. 21, n. 5, p. 652-62, 2007.

BRASIL. Ministério da Saúde. Manual de condutas para Úlceras Neurotróficas e traumáticas. Brasília: Ministério da Saúde, 2002. (Cadernos de Reabilitação em Hanseníase, 2). 
CHAVANTES, M. C.; VASEY, K. R.; KUALE, P. A. Influence of gaseous environment Laser tissue interaction in vivo. Lasers in Surgery and Medicine, New York, v. 8, p. 144-148, 1998.

CONLAN, M. J.; RAPLEY, J. W.; COBB, C. M. Biostimulation of wound healing by low-energy irradiation: A review. Journal of Clinical Periodontology, Copenhagen, v. 23, n. 5, p. 492-496, 1996.

CORAZZA, A. V. Fotobiomodulação comparativa entre o LASER e o LED de baixa intensidade na angiogênese de feridas cutâneas de ratos. 2005. Dissertação. (Mestrado em Bioengenharia) - Escola de Engenharia de São Carlos; Faculdade de Engenharia de Ribeirão Preto; Instituto de Química de São Carlos, São Carlos.

CORRÊA, F. I. Comparação do efeito antiinflamatório dos lasers de baixa potência GaAs (904 nm) e AlGaAs (630-670 nm) em modelo experimental de edema de pata em ratos e peritonite em camundongos induzidos por carragenina LPS e 48/80. 2003. Dissertação. (Mestrado em Engenharia Biomédica) - Universidade do Vale do Paraíba, São José dos Campos.

DIAS, M. D. Aplicações clínicas do oxigênio hiperbárico. Diagnóstico e Tratamento, São Paulo, v. 6, n. 1, p. 7-10, 2001.

FRADE, M. A.; CURSI, I. B.; ANDRADE, F. F.; SOARES, S. C.; RIBEIRO, W. S.; SANTOS, S. V.; FOSS, N. T. Úlcera de perna: um estudo de caso em Juiz de Fora - MG (Brasil) e região. Anais Brasileiros de Dermatologia, Rio de Janeiro, v. 80, n. 1, p. 41-46, 2005.

FRANKS, P. J.; MOODY, M.; MOFFATT, C. J.; HISKETT, G.; GATTO, P.; DAVIES, C.; FURLONG, W. T.; BARROW, E.; THOMAS, H. Randomized trial of two foam dressings in the management of chronic venous ulceration. Wound Repair and Regeneration, Malden, v. 15, n. 2, p. 197-202, 2007.

GAIDA, K.; KOLLER, R.; ISLER, C.; AYTEKIN, O.; AL-AWAMI, M.; MEISSL, G.; FREY, M. Low level laser therapy-a conservative approach to the burn scar? Burns: Journal of the International Society for Burn Injuries, Amsterdam, v. 30, n. 4, p. 362-367, Jun. 2004.

GUARNERA, G.; TINELLI, G.; ABENI, D.; DI PIETRO, C.; SAMPOGNA, F.; TABOLLI, S. Pain and quality of life in patients with vascular leg ulcers: an Italian multicentre study. Journal of Wound Care, London, v. 16, n. 8, p. 347-351, 2007.

HERBER, O. R.; SCHNEPP, W.; RIEGER, M. A. A systematic review on the impact of leg ulceration on patients' quality of life. Health and Quality of Life Outcomes, London, v. 5, p. 44, 2007.
JENSEN， I.; HARMS-RINGDAHL， K. Strategies for prevention and management of musculoskeletal conditions: Neck pain. Best Practice \& Research: Clinical Rheumatology, Amsterdam, v. 21, n. 1, p. 93-108, 2007.

JORGENSEN, B.; FRIIS, G. J.; GOTTRUP, F. Pain and quality of life for patients with venous leg ulcers: proof of concept of the efficacy of Biatain-Ibu, a new pain reducing wound dressing. Wound Repair and Regeneration, Malden, v. 14, n. 3, p. 233-239, 2006.

KARU, T. I.; KOLYAKOV, S. F. Exact Action Spectra for Cellular Responses Relevant to Phototherapy. Photomedicine and Laser Surgery, Larchmont, v. 23, n. 4, p. 355-361, 2005.

KARU, T. I.; KALENDO, G. S.; LETOKHO, V. V. S.; LOBKO, V. V. Biological action of low-intensity visible light on HeLa cells as a function of the coherence, dose, wavelength, and irradiation dose. Soviet Journal of Quantum Electronics, New York, v. 12, n. 9, p. 11341138, 1982.

Biological action of low-intensity visible light

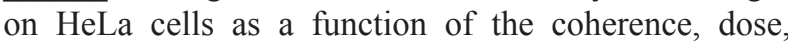
wavelength and irradiation regime. II. Sov. Journal Quantum Electronics, New York, v. 13, n. 9, p. 11691172, 1983.

KARU, T. I. Photobiological fundamentals of LowPower therapy. IEEE Journal of Quantum Electronics, New York, v. 23, n. 10, p. 1703-1717, 1987.

LAGAN, K. M.; MC DONOUGH, S. M.; CLEMENTS, B. A.; BAXTER, G. D. A case report of low intensity laser therapy (LILT) in the management of venous ulceration: potential effects of wound debridement upon efficacy. Journal of Clinical LaserMedicine \& Surgery, New York, v. 18, n. 1, p. 15-22, Feb. 2000.

LAGAN, K. M. etal. Clinical assessment and measurement of lower limb wounds: an intrarater reliability study. Irish Journal of Medical Science, Dublin, v. 168, n., p. 295-, 1999.

LAGAN, K. M.; CLEMENTS, B. A.; MCDONOUGH, S.; BAXTER, G. D. Low intensity laser therapy (830nm) in the management of minor postsurgical wounds: a controlled clinical study. Lasers in Surgery and Medicine, New York, v. 28, n. 1, p. 27-32, 2001.

LAGAN, K. M.; MCKENNA, T.; WITHEROW, A.; JOHNS, J.; MCDONOUGH, S. M.;BAXTER, G. D. LowIntensity Laser Therapy/Combined Phototherapy in the Management of Chronic Venous Ulceration: A PlaceboControlled Study. Journal of Clinical LaserMedicine \& Surgery, New York, v. 20, n. 3, p. 109-116, 2002. 
LAM, L. K.; CHEING, G. L. Effects of 904-nm low-level laser therapy in the management of lateral epicondylitis: a randomized controlled trial. Photomedicine Laser Surgery, Larchmont, v. 25, n. 2, p. 65-71, 2007.

MAFFEI, F. H. A.; LASTORIA, S; BONETTI, W. B; ROLLO, H. A; GIANNINI, M; MOURA, R. Doenças vasculares periféricas. 3. ed. Rio de Janeiro: Medsi, 2002. v. 2.

MARQUES, C. R. S.; MARTIN, A. A.; LIMA, C. J.; CONRADO, L. A. L.; SILVEIRA, F. L.; CARVALHO, M. V. The use of hyperbaric oxygen therapy and LEDtherapy in diabetic foot. Journal Proceedings of the SPIE, Bellingham, v. 5312, p. 47-53, 2004.

REDDY, G. K. Aplicações biomédicas do laser de baixa potência. In: DAVIS, C. M. Fisioterapia e reabilitação: terapias complementares. 2. ed. Rio de Janeiro: Guanabara Koogan, 2006. p. 407-424.

SCHUBERT, E. F. Light emitting diodes. 2. ed. Cambridge: Cambridge Univestity Press, 2006.

STERGIOULAS, A. Low-power laser treatment in patients with frozen shoulder: preliminary results. Photomedicine Laser Surgery, Larchmont, v. 26, n. 2, p. 99-105, 2008.

TAKAHASHI, Y.; HITOMI, S.; HIRATA, T.; FUKUSE, T.; YAMAZAKI, F.; CHO, K.; WADA, H. Neovascularization effect with $\mathrm{He}-\mathrm{Ne}$ laser in the rat trachea. The Journal of Thoracic and Cardiovascular Surgery, Saint Louis, v. 40, n. 5, p. 288-291, 1992.

THOMAZ, J. B. Úlceras nos membros inferiores: diagnóstico e terapêutica. São Paulo: Fundo Editorial BYK, 2002.
VINCK, E.; COOREVITS, P.; CAGNIE, B.; DE MUYNCK, M.; VANDERSTRAETEN, G.; CAMBIER, D. Evidence of changes in sural nerve conduction mediated by light emitting diode irradiation. Lasers in Medical Science, London, v. 20, n. 1, p. 35-40, 2005.

VINCK, E. M.; CAGNIE, B. J.; CORNELISSEN, M. J.; DECLERCQ, H. A.; CAMBIER, D. C. Increased fibroblast proliferation induced by light emitting diode and low power laser irradiations. Lasers in Medical Science, London, v. 18, n. 2, p. 95-99, 2003.

WHELAN, H. T.; BUCHMANN, E. V.; WHELAN, N. T.; TURNER, S. G.; CEVENINI, V.; STINSON, H.; IGNATIUS, R.; MARTIN, T.; CWIKLINSKI, J.; MEYER, G. A.; HODGSON, B.; GOULD, L.; KANE, M.; CHEN, G.; CAVINESS, J. NASA Light emitting diode medical applications from deep space to deep sea. AIP Conference Proceedings, New York, v. 552, n. 1, p. 35-45, Feb. 2001.

YU, W.; NAIM, J. O.; MCGOWAN, M.; IPPOLITO, K.; LANZAFAME, R. J. a) Photomodulation of oxidative metabolism and electron chain enzymes in rat liver mitochondria. Photochemistry and Photobiology, Oxford, v. 66, n. 6, 866-871, 1997.

YU, W.; NAIM J. O.; LANZAFAME R. J. b) Effects of photostimulation on wound healing in diabetic mice. Lasers in Surgery and Medicine, New York, v. 56, 1997. (não é et al.) 
